\title{
GAPS IN THE CLOUD COVER? COMPARING EXTINCTION MEASURES IN SPIRAL DISKS
}

\author{
B. W. Holwerda, ${ }^{1}$ M. Meyer, ${ }^{1}$ M. Regan, ${ }^{1}$ D. Calzetti, ${ }^{2}$ K. D. Gordon, ${ }^{3}$ J. D. Smith, ${ }^{3}$ \\ D. Dale, ${ }^{4}$ C. W. Engelbracht,${ }^{3}$ T. Jarrett, ${ }^{5}$ M. Thornley, ${ }^{6}$ C. Bot, ${ }^{7}$ \\ B. Buckalew, ${ }^{8}$ R. C. Kennicutt, ${ }^{9}$ and R. A. GonzÁlez ${ }^{10}$ \\ Received 2007 May 31; accepted 2007 July 19
}

\begin{abstract}
Dust in galaxies can be mapped either by the FIR/submillimeter emission, the optical or infrared reddening of starlight, or the extinction of a known background source. We compare two dust extinction measurements for a set of 15 sections in 13 nearby galaxies to determine the scale of the dusty interstellar medium (ISM) responsible for disk opacity: one using stellar reddening and the other a known background source. In our earlier papers, we presented extinction measurements of 29 galaxies, based on calibrated counts of distant background objects identified though foreground disks in Hubble Space Telescope WFPC2 images. For the 13 galaxies that overlap with the Spitzer Infrared Nearby Galaxies Survey, we now compare these results with those obtained from an $I-L$ color map. Our goal is to determine whether or not a detected distant galaxy indicates a gap in the dusty ISM, and hence to better understand the nature and geometry of the disk extinction. We find that distant galaxies are predominantly in lowextinction sections marked by the color maps, indicating that their number depends both on the cloud cover of Spitzerresolved dust structures, mostly the spiral arms, and a diffuse, unresolved underlying disk. We note that our infrared color map $[E(I-L)]$ underestimates the overall dust presence in these disks severely because it implicitly assumes the presence of a dust screen in front of the stellar distribution.

Key words: dust, extinction — galaxies: individual (NGC 925, NGC 2841, NGC 3198, NGC 3351, NGC 3621, NGC 3627, NGC 4321, NGC 4536, NGC 4559, NGC 5491, NGC 6946, NGC 7331) galaxies: ISM — galaxies: spiral — infrared: galaxies — infrared: ISM — ISM: structure
\end{abstract}

\section{INTRODUCTION}

The dust content of a spiral galaxy can be estimated using several different observational techniques: (1) observations of the dust's typical emission in the far-infrared and submillimeter spectral ranges (e.g., Dale et al. 2005; Meijerink et al. 2005); (2) the reddening and dimming effect on stellar populations in the UV (e.g., Boissier et al. 2004), optical (e.g., Elmegreen 1980), and infrared (e.g., Regan 2000; M. Meyer et al. 2007, in preparation); (3) the balancing of the disk's energy budget, which produces its spectral energy distribution (SED; e.g., Cortese et al. 2006; Popescu \& Tuffs 2005); or (4) the absolute absorption of a known background source (e.g., Domingue et al. 1999; González et al. 1998; Elmegreen et al. 2001; Holwerda 2005). Reviews of the various approaches can be found in Calzetti (2001), Popescu \& Tuffs (2005), Tuffs \& Popescu (2005), and references therein.

\footnotetext{
1 Space Telescope Science Institute, 3700 San Martin Drive, Baltimore, MD 21218, USA.

2 Department of Astronomy, University of Massachusetts, 710 North Pleasant Street, Amherst, MA 01003, USA.

3 Steward Observatory, University of Arizona, 933 North Cherry Avenue, Tucson, AZ 85721, USA.

4 Department of Physics and Astronomy, University of Wyoming, Laramie, WY 82071, USA.

5 Spitzer Science Center, California Institute of Technology, MS 220-6, 1200 East California Boulevard, Pasadena, CA 91125, USA.

6 Bucknell University, Lewisburg, PA 17837, USA.

${ }^{7}$ California Institute of Technology, MC-320-47, 1200 East California Boulevard, Pasadena, CA 91125, USA.

8 Department of Physics, Embry-Riddle University, 3700 Willow Creek Road, Prescott, AZ 86301, USA.

9 Institute of Astronomy, University of Cambridge, Madingley Road, Cambridge CB3 0HA, UK

${ }^{10}$ Centro de Radiastronomía y Astrofísica, Universidad Nacional Autónoma de México, 58190 Morelia, Michoacán, Mexico.
}

All of these techniques have different and specific strengths and weaknesses, and hence a comparison between the results of two such techniques offers insight into the nature of the dust in disks. In two recent papers (Holwerda et al. 2007a, 2007b), we compared the dust surface densities estimated from distant galaxy counts and the star/dust SED. Here, we focus on the comparison between extinction maps based on stellar reddening and results from the distant background object counts. The reddening map's advantage over the SED measurement is spatial resolution. The simple screen geometry we use here has been surpassed in extinction studies for some time (e.g., Elmegreen 1980; Kodaira \& Ohta 1994) but will suffice to determine the scale of the dusty interstellar medium (ISM) structure that is responsible for reducing the number of distant galaxies seen through a spiral disk. Our goal is to determine whether an observed distant galaxy indicates a hole in the ISM disk that would correspond to no extinction in the reddening map.

An independent extinction measurement requires a known background source other than the stellar disk. White \& Keel (1992) proposed an occulted elliptical galaxy, and González et al. (1998) used the calibrated number of distant galaxies seen through a foreground disk in a Hubble Space Telescope (HST) image. Both techniques have been used on nearby disks and have by now exhausted nearby occulting pairs (Andredakis \& van der Kruit 1992; Berlind et al. 1997; Domingue et al. 1999, 2000; White et al. 2000; Keel \& White 2001a, 2001b; Elmegreen et al. 2001) and HST WFPC2 images suitable for distant galaxy counts (Holwerda et al. 2005a, 2005b, 2005c, 2005d, 2005e). The known background source is either rare (the occulted large galaxy) or inherently uncertain due to cosmic variance (number density of distant background galaxies). The advantages are that no assumption about the dust/star geometry has to be made, and the absorption measurement is for the entire height of the disk. 
Models for the light of spiral disks have incorporated ever more complex geometries for extinction and reddening by dust, both for edge-on systems (e.g., Kodaira \& Ohta 1994; Xilouris et al. 1999; Kylafis \& Xilouris 2005) and face-on spirals (e.g., Elmegreen 1980; Elmegreen \& Block 1999; Disney et al. 1989; Rix \& Rieke 1993; Peletier et al. 1995). These have been used to correct stellar populations (Elmegreen 1980; Rix \& Rieke 1993) and star formation (see the review of Calzetti 2001 and references therein). The large-scale structure of the dusty ISM in spirals has been modeled this way with a dusty disk more extended but thinner than the stellar one (Elmegreen 1980; Peletier et al. 1995; Xilouris et al. 1999; Bianchi 2007). Dalcanton et al. (2004) argued that dust lanes are a sign of vertical instability in more massive disks, while smaller disks exhibit a fractured dust morphology. Recent observations point to a thicker or second vertical structure of dust ISM (Howk 1999; Howk \& Savage 1999; Thompson et al. 2004; Seth et al. 2005; Kamphuis et al. 2007).

Monte Carlo simulations of photons in the disk also use ever more sophisticated geometry: spherical (Witt et al. 1992; Witt \& Gordon 2000), cylindrical (Bianchi et al. 1996), planar (Bruzual A et al. 1988; Baes \& Dejonghe 2001a, 2001b), and, recently, arbitrary geometry (e.g., Gordon et al. 2001; Misselt et al. 2001; Baes et al. 2003). The clumpiness of the ISM has been proven to be the chief reason why the observed extinction law differs from the Galactic one (Natta \& Panagia 1984; Calzetti et al. 1994; Fischera et al. 2003; Fischera \& Dopita 2005). The aim of models has shifted to predicting not only the reddening and extinction by the dust in spirals, but also the contribution from the ISM's emission to the overall SED.

Multiwavelength models and observations of disks are addressing several questions regarding spiral disks: star formation measurements (e.g., Calzetti et al. 2005; Dopita et al. 2006b, 2006a), ISM composition (e.g., Draine \& Li 2001, 2007; Li \& Draine 2001; Draine et al. 2007), energy balance (Popescu et al. 2000; Misiriotis et al. 2001; Tuffs et al. 2004; Dasyra et al. 2005), and stellar populations (Dale et al. 2005, 2007).

The results from the Spitzer Infrared Nearby Galaxy Survey (SINGS; Kennicutt et al. 2003) have shed light on the relation between dust and star formation within a range of galaxy types and environments: the starburst galaxy M51 (Calzetti et al. 2005; Thornley et al. 2006), the grand-design spiral M81 (Gordon et al. 2004; Pérez-González et al. 2006), the rings of NGC 7331 (Regan et al. 2004) and M31 (Gordon et al. 2006), the superwind in M82 (Engelbracht et al. 2006), and the dwarf NGC 55 (Engelbracht et al. 2004). Comparative studies for the whole sample of galaxies can be found in Dale et al. $(2005,2007)$ and Draine et al. (2007).

The mid-infrared bands at 3.6 and $4.5 \mu \mathrm{m}$ are dominated by the emission from the older, smooth stellar disk; either combined with the $I$-band data can provide a very useful dust-screen extinction map (Regan 2000; M. Meyer et al. 2007, in preparation). We employ the simple screen approach because (1) in the near and mid-infrared we expect most of the disk to be optically thin, with the Spitzer bands providing the lowest-extinction view of the disk, and (2) we are only interested in relative opacity values.

The advantage of the color-based extinction map is that the spatial resolution is as high as the original single-band images; however, the derived extinction values are limited by the assumptions about geometry (a single screen of dust in front of a layer of stars) and stellar population (a single stellar color everywhere).

We want to know whether or not a disk is fully transparent in those places where a distant galaxy can be identified in HST images. The notion that those lines of sight are devoid of dust seems to be in contradiction with the picture of a ubiquitous, fractal, and clumpy medium that has developed since the discussions on the opacity of spiral disks (Davies \& Burstein 1995).

The organization of this paper is as follows: $\oint 2$ describes the data, $\S 3$ details the calibrations of the extinction maps, $\S 4$ briefly describes the distant galaxy counts, and $\S \S 5$ and 6 contain our discussion and conclusions.

\section{DATA}

In this paper we use the Spitzer IRAC mosaics, and accompanying ground-based $I$-band data, from the fifth data release of the SINGS project (Kennicutt et al. 2003, 2007). Part of the Spitzer data products are mosaics of the four IRAC channels with a pixel scale of $0.75^{\prime \prime}$ in $\mathrm{MJy} \mathrm{sr}^{-1}$, aligned with the sky coordinates. The mosaics are the product of a specialized pipeline to register and "drizzle" the basic calibrated data to a single mosaic.

The ground-based $I$-band images are a complementary SINGS data product. The world coordinates of the I-band data are updated using the stellar positions in the IRAC, channel $1(3.6 \mu \mathrm{m})$ mosaic. The $I$-band images are then shifted to the IRAC mosaic coordinates and degraded to the same resolution, estimated from the stars in the field, generally with a FWHM of $2.5^{\prime \prime}$.

\section{EXTINCTION MAPS}

The Spitzer IRAC channels 1 and 2 (3.6 and $4.5 \mu \mathrm{m}$ ) are mostly devoid of the spectral features of interstellar polycyclic aromatic hydrocarbons (PAH) and suffer little from extinction by the larger dust grains. In this paper we use channel $1(3.6 \mu \mathrm{m})$ to map the stellar emission. The $I-L$ color is proportional to the extinction in $I\left(A_{I}\right)$,

$$
A_{I}=a[E(I-L)+b] .
$$

This leaves two free parameters for the NIR extinction law: the zero point, $b$, and the slope, $a$. The slope of the IR extinction law is very close to unity: $a=0.88$ (Rieke \& Lebofsky 1985) for single lines of sight. The zero-point $b$ can be found using the IRAC $8 \mu \mathrm{m}$ PAH map of the same galaxy. ${ }^{11}$

To determine the zero point $(b)$ we find the 100 lowest-value pixels in the $8 \mu \mathrm{m}$ PAH map, after sigma clipping to remove the sky. The median value of the 100 corresponding pixels in the extinction map is our zero point $b$, i.e., the stellar color of the disk before extinction. Table 1 lists the zero-extinction colors for our maps.

The extinction maps are clipped to the $1 \sigma$ level calculated from the sky noise in both $I$ and $L$ images. The spatial resolution of the extinction maps is predominantly limited by the $I$-band observations; FWHM estimates of the point-spread function are around $2.5^{\prime \prime}$.

In the construction of the extinction maps, we make three significant assumptions: (1) the geometry of the dust and stars is a simple screen of dust in front of the stars; (2) regions lacking $8 \mu \mathrm{m}$ emission are indicative of the intrinsic stellar color; and (3) the $L$ band $(3.6 \mu \mathrm{m})$ is a good tracer of purely stellar emission. As noted in the introduction, more sophisticated models exist for the dust's effect on stellar emission, but these maps will suffice for our purposes. Similar maps are used by M. Meyer et al. (2007, in preparation) to trace the dusty ISM for comparison with the morphology of $\mathrm{CO}$ and $\mathrm{H}$ i maps.

\footnotetext{
11 The $8 \mu \mathrm{m}$ PAH-only map is obtained from IRAC's channel 4 by subtracting the stellar contribution to it. This contribution is estimated from channels 1 and 2 (3.6 and $4.5 \mu \mathrm{m}$ ), using the same formalism as Pahre et al. (2004a, 2004b) but with the improved aperture corrections from T. Jarrett (http://spider.ipac.caltech.edu/ staff/jarrett/irac/calibration/index.html) and Dale et al. (2007).
} 
TABLE 1

\begin{tabular}{|c|c|}
\hline Galaxy & $E(I-L)$ \\
\hline NGC $925 \ldots \ldots$ & -0.5 \\
\hline NGC $2841 \ldots \ldots$ & -0.3 \\
\hline 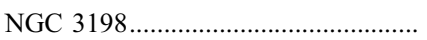 & -0.4 \\
\hline NGC $3351 \ldots \ldots \ldots$ & -1.2 \\
\hline NGC $3621 \ldots \ldots$ & -0.9 \\
\hline 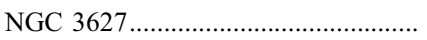 & -0.4 \\
\hline 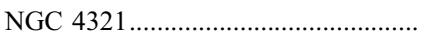 & -0.3 \\
\hline 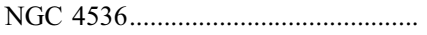 & -0.3 \\
\hline NGC $4559 \ldots \ldots \ldots \ldots$ & -0.4 \\
\hline NGC $4725 \ldots \ldots \ldots$ & -0.4 \\
\hline NGC 5194... & -0.6 \\
\hline 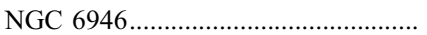 & -0.3 \\
\hline NGC 7331 & -0.3 \\
\hline
\end{tabular}

Note.-The values display a range not unreasonable for spiral disk stellar populations (Bell \& de Jong 2001).

The first assumption is a screen of dust in front of the stars. This is the simplest geometry one could use, and it allows the application of the extinction law as found for single lines of sight. Mixing the extincting dust and stars would result in a decoupling of the relation between color and extinction (Natta \& Panagia 1984; Calzetti et al. 1994; Fischera et al. 2003; Fischera \& Dopita 2005). Alternative maps could be constructed using a more gray relation than equation (1), reflecting the mix of dust and stars. Unfortunately, the choice of extinction law is problematic, as the exact mix and geometry are unknown and likely to change in different sections of the disk. However, we are interested in the relative extinction in our maps (whether or not distant galaxies are preferentially detected in low-extinction regions) and not in the absolute extinction along a given line of sight. We note that these maps represent lower limits for the total extinction through the disk.

The second assumption is that PAH emission does not arise in sections that have no interstellar dust; therefore, these sections will exhibit no reddening in the $E(I-L)$ map. Recent observations with Spitzer have found regions in galaxies with $24 \mu \mathrm{m}$ emission but no $8 \mu \mathrm{m}$ emission (e.g., Meixner et al. 2006). This is attributed to different processing of the dust grains and PAHs by the strong UV fields of star-forming regions. However, the lowest fluxes of $\mathrm{PAH}$ emission in these images occur not in the centers of bright star-forming regions but in the disk, between the arms.

The third assumption is the choice of reference band for the purely stellar emission. M. Meyer et al. (2007, in preparation) chose channel $2(4.5 \mu \mathrm{m})$ as the NIR reference, and in this paper we use channel $1(3.6 \mu \mathrm{m})$. Both suffer small-scale contamination from nonstellar emission; channel $2(4.5 \mu \mathrm{m})$ may contain hot dust emission ( Lu et al. 2003; Pahre et al. 2004a; Regan et al. 2004; Calzetti et al. 2005; Hunter et al. 2006), and channel $1(3.6 \mu \mathrm{m})$ comprises a smaller PAH emission feature, centered near $3.3 \mu \mathrm{m}$, and may contain hot dust emission as well. In both cases, the PAH or hot dust would increase the flux in the "stellar" channel, artificially elevating the implied extinction in the $I$ band. This additional uncertainty is on the order of the stellar color gradient (approximately a tenth of a magnitude). Both of the uncertainties, contamination and stellar gradient, are much smaller than the uncertainty due to the assumed geometry of the stars and dust in the extinction values in the presented maps, but they do change the distribution. To check for systematic effects between channel 1 and 2, we compared our extinction map of NGC 3627 made from channel 1 (3.6 $\mu \mathrm{m}$; Fig. 1) to one using channel $2(4.5 \mu \mathrm{m})$ from M. Meyer et al. (2007, in preparation, their Fig. 3). The comparison reveals little difference in structure (only some disparities in the $\mathrm{H}$ II regions in the spiral arms) and an overall offset of $0.05 \mathrm{mag}$ in extinction. Because the PAHs affect only $\mathrm{H}$ II regions and we are interested in the overall disk, this should not strongly influence our overall conclusions.

Given the above assumptions (the star/dust geometry, the intrinsic stellar color of the disk, and the link between lack of extinction and absence of $8 \mu \mathrm{m}$ PAH emission), the absolute calibration of the extinction maps is uncertain. The dominant presupposition is that of the geometry (and hence the extinction law), which most certainly results in an underestimate of the dust in these galaxies. However, because we investigate the "preference" of distant objects for low- or high-extinction regions, these maps will suffice to distinguish between sections of higher and lower extinction.

\section{DISTANT GALAXY COUNTS}

Holwerda et al. (2005b) presented disk-extinction measurements based on counts of distant galaxies, calibrated for crowding and confusion with the synthetic field method (SFM; González et al. 1998; Holwerda et al. 2005a). The calibration is done with a series of "synthetic fields," the original image with an artificially dimmed Hubble Deep Field (HDF) added to it. The relation between artificial dimming and HDF galaxies retrieved from the synthetic field can then be used to find the average extinction in the field from the number of observed distant galaxies found in the original field. The relation between the number of HDF galaxies and dimming is unique for each field, and synthetic fields are made for each science field. In the zero-extinction synthetic field, the added HDF galaxies show where distant galaxies could have been detected under the crowding conditions in the science field. The SFM gives an average opacity for the whole height of a section of the spiral disk. ${ }^{12}$

In Holwerda et al. (2005b) we already speculated that if the galaxies identified in the WFPC2 field are in those sections of the disk that are effectively transparent, then the average extinction found from the SFM is an indication of dust cloud cover. If so, we found that typical covering fractions are $40 \%$ for the disk and $60 \%$ for the spiral arms, assuming fully opaque clouds. We have compared these disk opacity measurements with those from the occulting galaxy technique (Holwerda et al. 2005b), and those provided by an SED model based on the IRAC and MIPS data (Holwerda et al. 2007a). The disk opacity valuesapparent optical depths - for all three methods agree well. In Holwerda et al. (2007a) we found a typical cloud optical depth of 0.4 , which corresponds to a typical cloud size of $60 \mathrm{pc}$.

In this paper, we explore the earlier assertion that distant galaxies are predominantly found in those sections of the disk that are completely transparent, something that appears somewhat contradictory to the small scale found in our comparison with the SED. Therefore, we quantify to what extent large dust clouds resolved with IRAC determine the SFM opacity value.

\section{DISTANT GALAXY NUMBER AND DISK EXTINCTION}

The $I$-band extinction maps based on the above method are presented in Figure 1. The extinction is higher in the spiral arms, and the effects of $\mathrm{H}$ II regions can clearly be seen. In Figure 1, the WFPC2 footprint and the positions of the identified distant galaxies from Holwerda et al. (2005b) are also plotted on the extinction

\footnotetext{
12 The term disk opacity is defined as the apparent optical depth for the whole height of the disk expressed in magnitudes.
} 

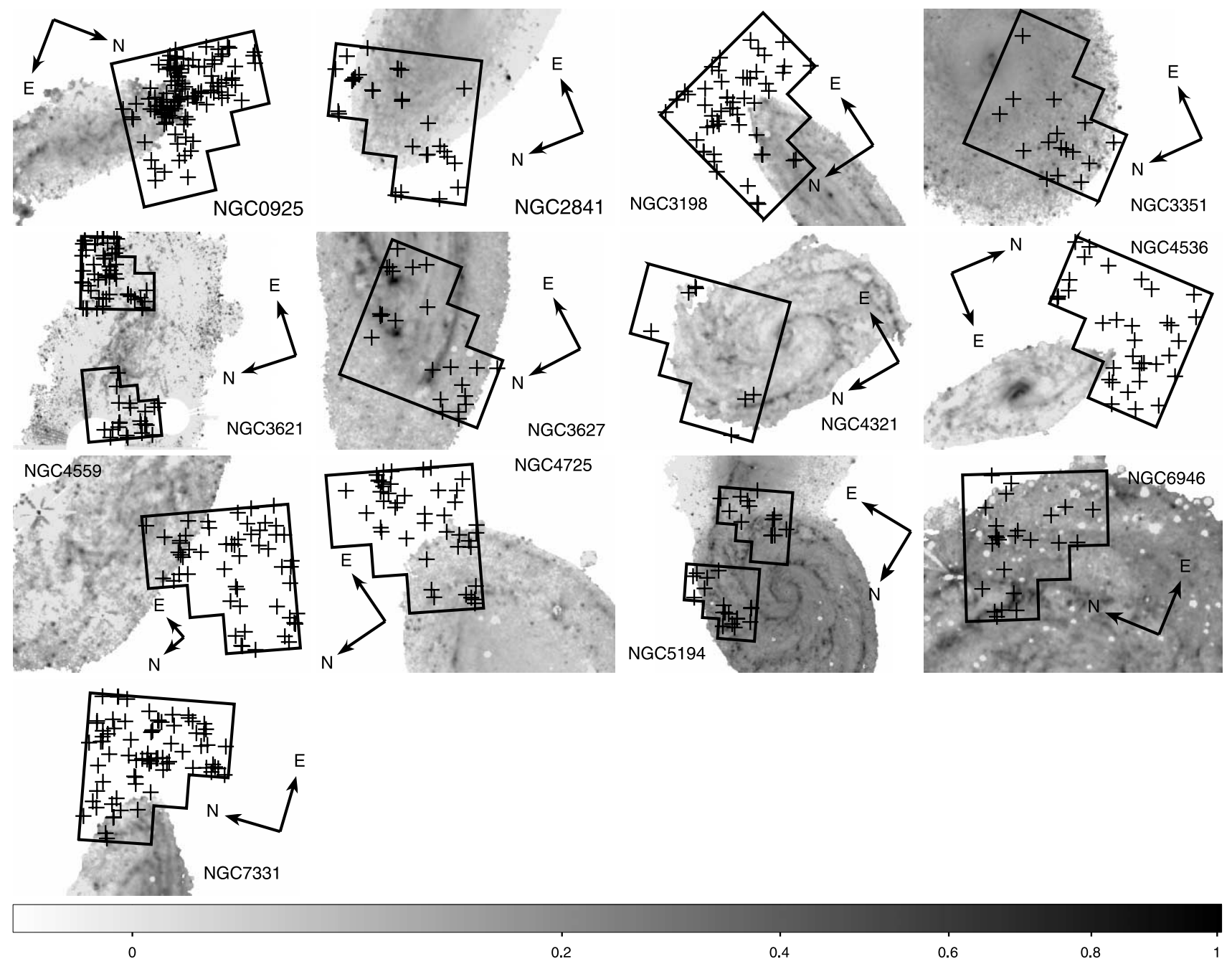

FIg. 1.-Details from the extinction maps, based on the $E(I-L)$ color, for the 13 SINGS galaxies in our sample. Gray-scale values are the optical depth $\tau$ implied by the $I-L$ color. The WFPC2 field of view used for the galaxy counts in Holwerda et al. (2005b) is overlaid. The crosses are the positions of the distant galaxies identified in the HST WFPC2 data.

map. Since the WFPC2 fields are centered on a spiral arm further out in the disk, the sigma-clipped extinction maps often do not cover the entire field of view of the WFPC $2 .{ }^{13}$

The distant galaxies identified by Holwerda et al. (2005b) appear to be mostly in the regions of lower extinction (Fig. 1). To test this, we compare the distribution of extinction values at the positions of observed distant background galaxies to the distribution of extinction values where a distant galaxy could be found, i.e., the position of the synthetic galaxies. For the reference distribution, we use the extinction values at the positions of the added HDF distant galaxies in the zero-extinction synthetic field. These are positions in the extinction map where we could have found a distant galaxy, given the crowding and confusion conditions in the HST WFPC2 images.

Figure 2 shows the cumulative histogram for these two sets of extinction values, at the position of the observed distant galaxies and the synthetic ones. The majority of the observed distant galaxies are detected predominantly below extinction values of $0.2 \mathrm{mag}$. Artificial, synthetic distant galaxies can be found in regions with larger extinction values. A Kolmogorov-Smirnov test shows that the probability that the two distributions are similar is

\footnotetext{
${ }^{13}$ In the case of NGC 3198 and NGC 4536, the overlap of the WFPC2 field and the extinction map is small (Fig. 1), and these two galaxies do not contribute much to the statistical result (Fig. 2).
}

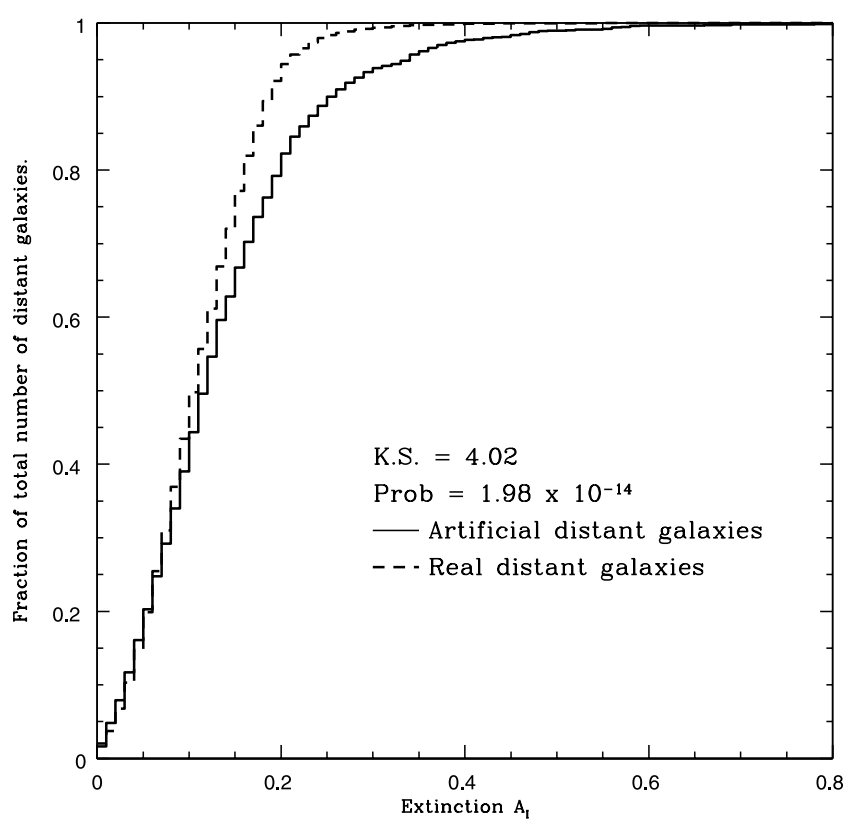

Fig. 2.-Cumulative histogram of extinction values at the position of observed distant background galaxies (dotted histogram), compared to the histogram of extinction values based on the positions of artificial galaxies within the WFPC2 field of view (solid histogram). 
TABLE 2

Average Apparent Extinction from the Number of Distant Galaxies, $A_{\mathrm{SFM}}$, The Spitzer SED, $A_{\mathrm{SED}}$, From Holwerda et AL. (2007), ANd the Color Maps in This Work, $A_{(I-L)}$

\begin{tabular}{|c|c|c|c|}
\hline Galaxy & $A_{\mathrm{SFM}}$ & $A_{\mathrm{SED}}$ & $A_{(I-L)}$ \\
\hline NGC $925 \ldots \ldots . .$. & $-0.4_{-0.3}^{+0.3}$ & 0.7 & $0.11 \pm 0.07$ \\
\hline NGC $2841 \ldots \ldots \ldots \ldots \ldots \ldots . . . . . .$. & $0.8_{-0.4}^{+0.5}$ & 1.5 & $0.08 \pm 0.05$ \\
\hline 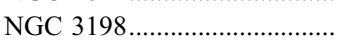 & $0.8_{-0.3}^{+0.4}$ & 0.8 & $0.11 \pm 0.07$ \\
\hline 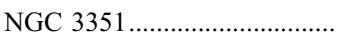 & $1.2_{-0.5}^{+0.6}$ & 1.1 & $\ldots$ \\
\hline 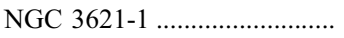 & $2.2_{-0.6}^{+0.6}$ & 1.1 & $0.08 \pm 0.08$ \\
\hline 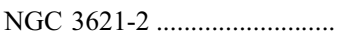 & $1.0_{-0.3}^{+0.0}$ & 0.8 & $0.09 \pm 0.10$ \\
\hline 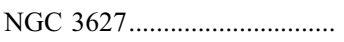 & $2.1_{-0.7}^{+0.7}$ & 1.8 & $0.12 \pm 0.11$ \\
\hline NGC $4321 \ldots \ldots \ldots \ldots . . .$. & $2.3_{-0.8}^{+0.8}$ & 3.0 & $0.06 \pm 0.06$ \\
\hline NGC $4536 \ldots \ldots \ldots \ldots \ldots \ldots \ldots \ldots \ldots \ldots$ & $0.9_{-0.4}^{+0.4}$ & 0.3 & $0.11 \pm 0.07$ \\
\hline 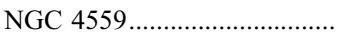 & $0.1_{-0.3}^{+0.3}$ & 0.3 & $0.08 \pm 0.06$ \\
\hline 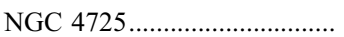 & $0.8_{-0.3}^{+0.3}$ & 0.5 & $0.05 \pm 0.05$ \\
\hline 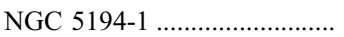 & $-0.4_{-0.4}^{+0.4}$ & 3.6 & $0.24 \pm 0.08$ \\
\hline NGC 5194-2 ............................ & $1.4_{-0.6}^{+0.6}$ & 4.2 & $0.18 \pm 0.09$ \\
\hline 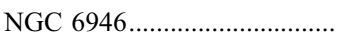 & $1.1_{-0.5}^{+0.6}$ & 1.4 & $0.18 \pm 0.11$ \\
\hline 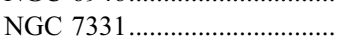 & $0.3_{-0.3}^{+0.3}$ & 0.5 & $0.11 \pm 0.08$ \\
\hline
\end{tabular}

Note.-These values have not been corrected for inclination, because such correction depends on the assumed dust geometry and the dust's effective filling factor.

negligible $\left(1.98 \times 10^{-14}\right)$. Hence, an observed distant galaxy is more likely to be found in a low-extinction section of the disk. $^{14}$

Cloud cover by optically thick clouds, which can be resolved with Spitzer, is one factor reducing the detected number of distant galaxies observed through a disk. However, distant background galaxies are not found exclusively at zero-extinction regions; $60 \%$ of the observed galaxies are identified at more than $0.1 \mathrm{mag}$ of extinction (see Fig. 2). Therefore, a second factor determining their observed number is the lower extinction, unresolved dust disk. If this is a cloud filling factor as well (discrete clouds rather than a screen), these are many smaller clouds in addition to the struc-

\footnotetext{
${ }^{14}$ The limiting factor in the detection of HDF galaxies is the "granularity" in the science field, i.e., how resolved the stellar disk is (see our discussion in González et al. 2003; Holwerda et al. 2005e), not the disk's opacity.
}

tures seen in the extinction maps, consistent with our result in Holwerda et al. (2007a).

How much of the opacity measured from missing distant galaxies is caused by complete blocking of the line of sight depends on what value in the extinction map from the infrared color translates into a distant galaxy that is unidentifiable as such. The extinction maps in Figure 1 underestimate the overall dust surface density and, hence, the opacity of the disks. This can be illustrated with a comparison between the average opacities (apparent optical depths) from the $E(I-L)$ extinction maps $\left(A_{(I-L)}\right)$, the overall Spitzer $\operatorname{SED}\left(A_{\mathrm{SED}}\right)$, and the number of distant galaxies $\left(A_{\mathrm{SFM}}\right)$. The average extinction values from the maps, from the part where there are data, are generally much lower than those derived from the SED or the number of distant galaxies (Table 2). This can partially be explained by the fact that the SFM measures the extinction for the entire height of the disk (the background sources are well beyond the disk) and the color map measures the extinction by at best half of the disk's height, which is the part backlit by the stellar disk. This would explain a factor of 2 difference between the two opacity values. The second reason that the extinction maps underestimate the total opacity is the assumed star/dust geometry. A mix of stars and dust does not follow a neat extinction law and will generally display a variety of extinction law behaviors, all of them more "gray" than the one from Rieke \& Lebofsky (1985) depending on geometry (as shown previously by several authors, notably Elmegreen 1980; Natta \& Panagia 1984). Let us set values in the $E(I-L)$ map that would be opaque for a distant galaxy and compare how the cloud cover explains the missing distant galaxies.

In our $E(I-L)$ extinction maps, $A_{(I-L)}=0.5$ (half the height of the disk), or possibly $A_{(I-L)}=0.3$ (a third of the height), is the naively expected opaque disk value. Table 3 lists the covering percentage of the extinction maps for pixels above $A_{(I-L)}=$ $0.5,0.3$, and 0.1 for the whole map and the section covered by the WFPC2. Extinction values greater than either 0.5 and 0.3 fail to cover the fraction implied by the SFM measurement. Only if the $A_{(I-L)}$ extinction is underestimated by a factor of 10 do the cloud cover fractions match up to the same order, yet we found most of our real observed distant galaxies at positions with $0.1 \mathrm{mag}$ of extinction (Fig. 2). Hence, if we take $A_{(I-L)} \approx 0.3$ as the point

TABLE 3

Cloud Covering Percentages of the Disk for Different Values of Disk Opacity $(\tau>0.5,0.3$, and 0.1$)$, Both for the Entire Extinction Map $\left(f_{\text {map }}\right)$, as Well as for the Section Covered by the WFPC2 $\left(f_{\text {Wfpc }}\right)$

\begin{tabular}{|c|c|c|c|c|c|c|c|}
\hline \multirow[b]{2}{*}{ GALAXY } & \multicolumn{3}{|c|}{$f_{\text {map }}$} & \multicolumn{3}{|c|}{$f_{\mathrm{WFPC}}$} & \multirow[b]{2}{*}{$f_{\mathrm{SFM}}$} \\
\hline & $>0.5$ & $>0.3$ & $>0.1$ & $>0.5$ & $>0.3$ & $>0.1$ & \\
\hline NGC 925 & 0.2 & 1.6 & 38 & 0.1 & 4.9 & 58 & $\ldots$ \\
\hline NGC $2841 \ldots \ldots \ldots$ & 0.0 & 0.1 & 28 & 0.0 & 0.4 & 47 & 52 \\
\hline NGC $3198 \ldots \ldots \ldots \ldots \ldots \ldots$ & 0.1 & 1.3 & 55 & 0.3 & 3.3 & 74 & 52 \\
\hline 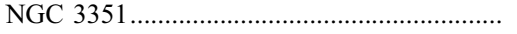 & 0.1 & 1.0 & 66 & 0.2 & 2.3 & 91 & 67 \\
\hline 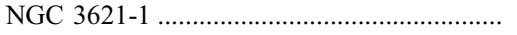 & 1.0 & 5.1 & 46 & 0.3 & 2.8 & 40 & 87 \\
\hline 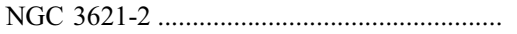 & 1.0 & 5.1 & 46 & 1.2 & 7.2 & 41 & 60 \\
\hline NGC $3627 \ldots \ldots$ & 0.5 & 3.4 & 39 & 2.4 & 8.2 & 72 & 86 \\
\hline 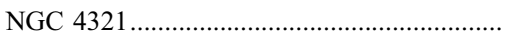 & 0.0 & 0.8 & 17 & 0.1 & 1.7 & 27 & 88 \\
\hline NGC 4536 & 0.7 & 2.7 & 17 & 0.0 & 3.9 & 61 & 56 \\
\hline NGC 4559 & 0.1 & 1.1 & 32 & 0.3 & 1.6 & 41 & 9 \\
\hline NGC $4725 \ldots$ & 0.0 & 0.1 & 10 & 0.0 & 0.5 & 22 & 52 \\
\hline 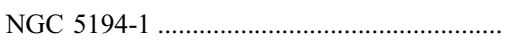 & 1.0 & 15 & 82 & 3.6 & 50 & 10 & $\ldots$ \\
\hline 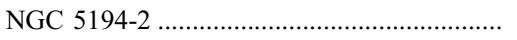 & 1.0 & 15 & 82 & 1.7 & 19 & 89 & 72 \\
\hline 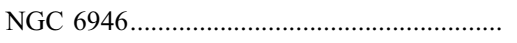 & 1.0 & 11 & 87 & 4.0 & 22 & 94 & 64 \\
\hline NGC 7331 & 0.4 & 10 & 61 & 0.4 & 7.5 & 64 & 24 \\
\hline
\end{tabular}

NoтE.-The column marked " SFM" $_{\mathrm{SF}}$ gives the cloud cover for the WFPC2 section implied from the SFM measurement, assuming only optically thick clouds. 
where the disk is opaque for distant galaxies, the big opaque clouds only block $\sim 10 \%$ of the typical background of distant galaxies outright (Fig. 2, solid line). That corresponds to $\sim 0.3 \mathrm{mag}$ of the SFM opacity measurement. Hence the remainder-often the majority - of the measured opacity is due to the unresolved and semitransparent disk.

In Holwerda et al. (2005b) we voiced our suspicion that the distant galaxies are detected in gaps in the ISM disk, based on the average color of these galaxies, which remained constant with the disk opacity inferred from their number. In addition, we found that the opacity from the counts of distant galaxies depended little on the inclination of the foreground disk.

This result we initially explained by a flat distribution of clouds for which the projected filling factor remains constant. This model did not specify the typical size of the opaque clouds, but a similar or greater angular size than the projected distant galaxies was implied. In Holwerda et al. (2007a) we found that small, optically thin clouds dominate the disks, using extra information from the Spitzer SED. Figure 2 appears to corroborate the hypothesis that the filling factor of large, resolved clouds do play a role in determining the number of distant galaxies found, but they are not the sole cause for their diminishing number and hence extinction in the disk. The second factor is the underlying disk of unresolved, small clouds. Therefore, the presence of a distant galaxy seen through a foreground disk does not imply that no dusty ISM is present along that particular line of sight.

\section{CONCLUSIONS}

From the extinction values at the position of distant galaxies we draw the following conclusions:

1. Distant galaxies are identified through a spiral disk in the lower opacity regions. Their number is, only in part, determined by the cloud cover of large, resolved clouds in the disk (Fig. 2).
2. Most of the distant galaxies are identified in parts of the disk with some extinction. Dusty ISM clouds, unresolved by Spitzer, are the second reason for the diminishing of their number (Fig. 2).

3. The apparent optical depth from the number of distant galaxies can be expressed as a cloud cover fraction; however, this implies opaque clouds, while the majority of the dust clouds are optically thin and unresolved.

4. We reconfirm that extinction maps from an infrared color [e.g., $E(I-L)]$ will mark the sections of higher extinction but underestimate the total extinction in any given part of the disk (Table 2) because of the intrinsic assumption of a simple geometry.

In future applications of the counts of distant galaxies as a probe of disk opacity, the nature of the cloud geometry, expressed as a cloud covering factor, should become increasingly evident. We hope to learn more about the nature of the smooth extinction disk in our future studies using distant galaxies as an extinction test in the HST ACS surveys of M51, M101, and M81.

The authors would like to thank T. Jarrett, for making his aperture corrections available, and B. Sugerman, for his help with the construction of the extinction maps. The authors would like to thank the referee, D. Elmegreen, for her comments; they helped tremendously to improve the paper. This work is based in part on archival data obtained with the Spitzer Space Telescope, which is operated by the Jet Propulsion Laboratory, California Institute of Technology, under a contract with NASA. This work is also based on observations with the NASA/ESA Hubble Space Telescope, obtained at the STScI, which is operated by the Association of Universities for Research in Astronomy (AURA), Inc., under NASA contract NAS5-26555. Support for this work was provided by NASA through grant number c3886 to D. Calzetti.
Andredakis, Y. C., \& van der Kruit, P. C. 1992, A\&A, 265, 396

Baes, M., \& Dejonghe, H. 2001a, MNRAS, 326, 722 2001b, MNRAS, 326, 733

Baes, M., et al. 2003, MNRAS, 343, 1081

Bell, E. F., \& de Jong, R. S. 2001, ApJ, 550, 212

Berlind, A. A., Quillen, A. C., Pogge, R. W., \& Sellgren, K. 1997, AJ, 114, 107

Bianchi, S. 2007, A\&A, in press

Bianchi, S., Ferrara, A., \& Giovanardi, C. 1996, ApJ, 465, 127

Boissier, S., Boselli, A., Buat, V., Donas, J., \& Milliard, B. 2004, A\&A, 424, 465

Bruzual A., G., Magris, G., \& Calvet, N. 1988, ApJ, 333, 673

Calzetti, D. 2001, PASP, 113, 1449

Calzetti, D., Kinney, A. L., \& Storchi-Bergmann, T. 1994, ApJ, 429, 582

Calzetti, D., et al. 2005, ApJ, 633, 871

Cortese, L., et al. 2006, ApJ, 637, 242

Dalcanton, J. J., Yoachim, P., \& Bernstein, R. A. 2004, ApJ, 608, 189

Dale, D. A., et al. 2005, ApJ, 633, 857 2007, ApJ, 655, 863

Dasyra, K. M., Xilouris, E. M., Misiriotis, A., \& Kylafis, N. D. 2005, A\&A, 437,447

Davies, J. I., \& Burstein, D. 1995, The Opacity of Spiral Disks (Dordrecht: Kluwer)

Disney, M., Davies, J., \& Phillipps, S. 1989, MNRAS, 239, 939

Domingue, D. L., Keel, W. C., Ryder, S. D., \& White, R. E. 1999, AJ, 118, 1542

Domingue, D. L., Keel, W. C., \& White, R. E. 2000, ApJ, 545, 171

Dopita, M. A., et al. 2006a, ApJS, 167, 177

- 2006b, ApJ, 647, 244

Draine, B. T., \& Li, A. 2001, ApJ, 551, 807

- 2007, ApJ, 657, 810

Draine, B. T., et al. 2007, ApJ, 663, 866

Elmegreen, B. G., \& Block, D. L. 1999, MNRAS, 303, 133

Elmegreen, D. M. 1980, ApJS, 43, 37

Elmegreen, D. M., Kaufman, M., Elmegreen, B. G., Brinks, E., Struck, C., Klarić, M., \& Thomasson, M. 2001, AJ, 121, 182

\section{REFERENCES}

Engelbracht, C. W., et al. 2004, ApJS, 154, 248

. 2006, ApJ, 642, L127

Fischera, J., \& Dopita, M. 2005, ApJ, 619, 340

Fischera, J., Dopita, M. A., \& Sutherland, R. S. 2003, ApJ, 599, L21

González, R. A., Allen, R. J., Dirsch, B., Ferguson, H. C., Calzetti, D., \& Panagia, N. 1998, ApJ, 506, 152

González, R. A., Loinard, L., Allen, R. J., \& Muller, S. 2003, AJ, 125, 1182

Gordon, K. D., Misselt, K. A., Witt, A. N., \& Clayton, G. C. 2001, ApJ, 551, 269

Gordon, K. D., et al. 2004, ApJS, 154, 215 2006, ApJ, 638, L87

Holwerda, B. W. 2005, Ph.D. thesis, Kapteyn Astronomical Institute

Holwerda, B. W., Draine, B., Gordon, K. D., González, R. A., Calzetti, D., Thornley, B., Allen, R. J., \& van der Kruit, P. C. 2007a, AJ, 134, in press

Holwerda, B. W., González, R. A., Allen, R. J., \& van der Kruit, P. C. 2005a, AJ, 129, 1381 2005b, AJ, 129, 1396 2005c, A\&A, 444, 101 2005d, A\&A, 444, 319

Holwerda, B. W., González, R. A., van der Kruit, P. C., \& Allen, R. J. 2005e, A\&A, 444, 109

Holwerda, B. W., et al. 2007b, in ASP Conf. Ser., Infrared Diagnostics of Galaxy Evolution, ed. R.-R. Chary (San Francisco: ASP), in press (astro-ph/0603395) Howk, J. C. 1999, Ap\&SS, 269, 293

Howk, J. C., \& Savage, B. D. 1999, AJ, 117, 2077

Hunter, D. A., Elmegreen, B. G., \& Martin, E. 2006, AJ, 132, 801

Kamphuis, P., Holwerda, B. W., Allen, R. J., Peletier, R. F., \& van der Kruit, P. C. 2007, A\&A, 471, L1

Keel, W. C., \& White, R. E. 2001a, AJ, 122, 1369 . 2001b, AJ, 121, 1442

Kennicutt, R. C., Jr., et al. 2003, PASP, 115, 928 2007, The Spitzer Infrared Nearby Galaxies Survey Fifth Data Delivery User's Guide (Pasadena: Caltech), http://data.spitzer.caltech.edu/popular/sings/ 20070410_enhanced_v1/Documents/sings_fifth_delivery_v2.pdf 
Kodaira, K., \& Ohta, K. 1994, PASJ, 46, 155

Kylafis, N. D., \& Xilouris, E. M. 2005, in AIP Conf. Proc. 761, The Spectral Energy Distributions of Gas-Rich Galaxies: Confronting Models with Data, ed. C. C. Popescu \& R. J. Tuffs (New York: AIP), 3

Li, A., \& Draine, B. T. 2001, ApJ, 554, 778

Lu, N., et al. 2003, ApJ, 588, 199

Meijerink, R., Tilanus, R. P. J., Dullemond, C. P., Israel, F. P., \& van der Werf, P. P. 2005 , A\&A, 430, 427

Meixner, M., et al. 2006, AJ, 132, 2268

Misiriotis, A., Popescu, C. C., Tuffs, R., \& Kylafis, N. D. 2001, A\&A, 372, 775

Misselt, K. A., Gordon, K. D., Clayton, G. C., \& Wolff, M. J. 2001, ApJ, 551, 277

Natta, A., \& Panagia, N. 1984, ApJ, 287, 228

Pahre, M. A., Ashby, M. L. N., Fazio, G. G., \& Willner, S. P. 2004a, ApJS, 154, 235 2004b, ApJS, 154, 229

Peletier, R. F., Valentijn, E. A., Moorwood, A. F. M., Freudling, W., Knapen, J. H., \& Beckman, J. E. 1995, A\&A, 300, L1

Pérez-González, P. G., et al. 2006, ApJ, 648, 987

Popescu, C. C., Misiriotis, A., Kylafis, N. D., Tuffs, R. J., \& Fischera, J. 2000, A\&A, 362, 138

Popescu, C. C., \& Tuffs, R. J. 2005, in AIP Conf. Proc. 761, The Spectral Energy Distributions of Gas-Rich Galaxies: Confronting Models with Data, ed. C. C. Popescu \& R. J. Tuffs (New York: AIP), 155
Regan, M. W. 2000, ApJ, 541, 142

Regan, M. W., et al. 2004, ApJS, 154, 204

Rieke, G. H., \& Lebofsky, M. J. 1985, ApJ, 288, 618

Rix, H., \& Rieke, M. J. 1993, ApJ, 418, 123

Seth, A. C., Dalcanton, J. J., \& de Jong, R. S. 2005, AJ, 130, 1574

Thompson, T. W. J., Howk, J. C., \& Savage, B. D. 2004, AJ, 128, 662

Thornley, M. D., Braine, J., \& Gardan, E. 2006, ApJ, 651, L101

Tuffs, R. J., \& Popescu, C. C. 2005, in AIP Conf. Proc. 761, The Spectral Energy Distributions of Gas-Rich Galaxies: Confronting Models with Data, ed. C. C. Popescu \& R. J. Tuffs (New York: AIP), 344

Tuffs, R. J., Popescu, C. C., Völk, H. J., Kylafis, N. D., \& Dopita, M. A. 2004, A\&A, 419, 821

White, R. E., \& Keel, W. C. 1992, Nature, 359, 129

White, R. E., Keel, W. C., \& Conselice, C. J. 2000, ApJ, 542, 761

Witt, A. N., \& Gordon, K. D. 2000, ApJ, 528, 799

Witt, A. N., Thronson, H. A., \& Capuano, J. M. 1992, ApJ, 393, 611

Xilouris, E. M., Byun, Y. I., Kylafis, N. D., Paleologou, E. V., \& Papamastorakis, J. 1999, A\&A, 344, 868 